\title{
The caregiving perspective in heart failure: a population based study
}

\author{
Patricia M Davidson ${ }^{1,5^{*}}$, Amy P Abernethy ${ }^{2}$, Phillip J Newton ${ }^{1}$, Katherine Clark ${ }^{3}$ and David C Currow ${ }^{4}$
}

\begin{abstract}
Background: Heart failure (HF) is a frequent condition in the elderly and mortality is high. This study sought to describe the profile of those providing care in the community and their needs.

Methods: The South Australian Health Omnibus is an annual, random, face-to-face, cross sectional survey conducted within the state. Having standardized data to the whole population, the study describes the subset of the population who identify that they actively cared for someone at the end of life with $\mathrm{HF}$ in the five years before survey administration.

Results: Three hundred and seventy three respondents (2.0\% of the whole population; $4.9 \%$ of caregivers) reported being a caregiver of someone with HF. There were 84 active caregivers (day-to-day or intermittent hands on caregivers) for people with HF. Mean age for caregivers for those with HF was much higher than other caregivers (55.7 vs 49.4; $p<0.001$ ) with care lasting for an average of 48.9 months (SD 66.2). People caring for those with HF were far less likely to access specialist palliative care services (38.1\% vs 60.9\%; $p<0.0001)$ despite having much greater levels of unmet needs for physical care $28.3 \%$ vs $14.1 \% ; p=0.008$ ).

Conclusion: Study findings suggest that there is a significant burden placed on caregivers for people with HF over extended periods in the community. There are differences in access to services for these caregivers compared to those dying from other conditions, particularly cancer.
\end{abstract}

Keywords: Heart failure, Caregivers, Australia

\section{Background}

Heart failure (HF) is a chronic illness with a poor prognosis $[1,2]$. In the elderly, caregiving needs are often accentuated and support systems are not always as readily accessible [3]. Increasingly there is interest in developing models of care to support individuals and their families who face dying with HF $[1,4,5]$. The impact of HF on individuals and health care systems are well described, yet much less is known about the impact of caregiving on informal caregivers, who are commonly family members [6,7]. The physical, psychological and social demands of caregiving can impact adversely on the well-being of caregivers [8]. Hwang and colleagues have noted that care provided for individuals with HF is much more extensive than that provided with usual spousal assistance and support [9].

\footnotetext{
* Correspondence: patriciamary.davidson@uts.edu.au

${ }^{1}$ Centre for Cardiovascular and Chronic Care, Faculty of Health, University of Technology Sydney, PO Box 123, Level 7, Building 10 Jones Street, Broadway, Sydney, NSW 2007, Australia

${ }_{5}^{5}$ School of Nursing, Johns Hopkins University, 525 N. Wolfe Street, Baltimore MD 21205, USA

Full list of author information is available at the end of the article
}

The increasing emphasis on community based HF management is placing additional pressure on family caregivers [10]. As a consequence, there is an increased stress on carers to provide physical care as well as social and emotional support. As in many other chronic conditions, caregiving has benefits and burdens: on the one hand it represents an opportunity for increased intimacy and connection, yet is also associated with significant burden, constancy of role, poor health outcomes for the caregiver and distress [6]. This is particularly the case if the carer is a spouse or in poor health themselves [11]. Consequently, there is a need to document and explore the role of caregiving in HF in the community [12]. Although there are some universally shared characteristics of caregiving, there are potentially some discrete differences among diagnostic groups of the care recipient. Although the merit of focussing on variable illness trajectories has been challenged [13], there is merit in understanding the extent of unmet needs and comparison of health service utilisation between different caregivers for different diagnoses.

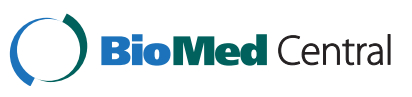


Although there has been extensive research on caregivers in many other clinical setting, research is limited in CHF and there are sparse recommendations to address unmet needs for support [14]. Available data suggests that caregiver burden increases if the caregiver experiences poor mental and/or physical health and has limited social and professional support [15]. Patients who are enrolled in specialty disease management programs are likely to have a level of caregiver support whereas those in primary care settings are less likely to have this support underscoring the need to undertake a population based approach to assessing needs.

Current guidelines recommendations suggest that interventions to reduce caregiver burden should focus on improving social support as well as control over their situation [16-18]. A comparison of caregivers with cancer, chronic obstructive pulmonary disease and HF demonstrated that caregiver resources not patient diagnosis or illness severity were associated with caregiver burden [19].

The majority of the data involving caregiver is focussed in the hospital and the immediate post discharge period [20], little is known about informal community caregiving in HF, particularly in the period leading to death. In Australia, the average time from referral to a palliative care service to death is 102 days, with $28.5 \%$ of people accessing specialist palliative care for longer than six months nationally [21]. In parallel with international trends, the proportion of those people with non-malignant conditions accessing specialist palliative care services is increasing.

This study describes the role of caregivers of people with HF from a population perspective, not simply those who access specialist or tertiary services [21]. This is more likely to generate a real world perspective of the increasing numbers of people providing care in the community.

Using a population-based approach, the aim of this study was to describe the characteristics of caregivers for people with HF at the end of life and compares these with other caregivers [21]. We have previously reported the findings of the survey related to met and unmet needs, [22] short- and long-term needs [23] and the relationship between perceived comfort and accessing palliative care services [22].

\section{Methods}

A random annual population-based health survey, the South Australian Health Omnibus Survey, has been conducted annually since 1991 [24]. An omnibus survey is a method of quantitative research using a stratified sample where data on a wide variety of subjects is collected during the same interview. Multiple researchers contribute individual questions while sharing the common demographic data collected from each respondent [21]. The Omnibus Survey is a face-to-face, cross-sectional survey. The survey is administered by a commercial research organisation with government support. There is a cost to researchers for each question included in the survey. Since 2000, information has been collected about the experiences of respondents who had someone close to them die from a life-limiting illness in the five years before responding [21]. A detailed response of the question route is describe elsewhere [23]. The questions are designed to access information relating to the nature of the respondent's relationship with the deceased person, the extent of their involvement in caregiving, their perception of needs (met or unmet), the use of palliative care and other services, and the main reason if a palliative care service was not used. For example caregiving was defined for the respondent as follows "Care' includes attention to any of the needs of the person, including hands-on care, overnight care, respite, shopping, collection of medications, taking to appointments, emotional support, bathing, etc." To incorporate differing levels of caregiver burden into the analysis, respondents were asked if they provided: "day-to-day hands-on care" (care 5-7 days per week); "intermittent hands-on care" (care 2-4 days per week); or, "rare hands-on care" (care 1 or less days per week). Pilot testing of the questionnaire with 50 members of the general public for comprehension and usability occurred each year and no changes were made to wording as a result of piloting. For the purposes of this study, the carers who identified themselves were compared to the remaining sample of caregivers, the majority of who reported deaths from cancer or end-stage respiratory disease.

\section{Sampling schema}

From September to December (2001-2007 inclusive), more than 4500 properties were approached annually to participate. In metropolitan areas, a starting point was randomly selected for a selection of 341 Australian Bureau of Statistics collector's district. In non-metropolitan areas, households were selected using 100 starting points statewide; all towns with a population greater than 10,000 were included and towns above 1000 were randomly included with chance of inclusion proportional to the size of the town. From the randomly selected starting point within each collector's district, 10 dwellings were selected using a skip pattern of every fourth household in a pre-arranged algorithm. A cluster size of 10 was used at each of the non-metropolitan starting points. One interview per household was conducted with the person over the age of 15 who had most recently had a birthday. If that person declined, no data were collected from that household. Interviews were conducted face-to-face by trained interviewers. Prompt cards were provided for selected answers to allow responses to be categorised. Data were entered using double entry techniques. Any missing responses were followed up by telephone. For quality assurance, 10\% 
of each interviewer's respondents were randomly selected and recontacted to confirm eligibility and responses. Aggregated data were then de-identified. These processes apply to the whole survey, are unchanged since the survey's inception in 1991, and could not be modified just for the questions relating to the end of life due to the Omnibus study methodology and the capacity to add limited items to the overall study structure. In spite of this limitation, the capacity to obtain a population based approach to caregiving at the end-of-life for individuals with HF is an important opportunity.

\section{Setting}

Australia supports a system of universal healthcare insurance which may be supplemented by private health insurance. Palliative care services can be accessed concurrently with other specialist services [25].

South Australia (SA) has a population of 1.54 million people [26]. Specialist palliative care services within SA span a range of different service delivery models, from large regional multidisciplinary teams within the capital city to single clinical nurses in small rural locations. Each service covers a geographic region, encompassing public and private hospitals, free-standing palliative care units, outpatient clinics and a community care team working in conjunction with general practitioners and community nurses. Almost $60 \%$ of people with a lifelimiting illness are referred to specialist palliative care services in SA [21]. There were five metropolitan and 14 rural/regional publicly funded specialist multidisciplinary palliative care services. The primary eligibility criterion to access these services is a life-limiting illness, irrespective of diagnosis or prognosis. These services are comprised of a team of multidisciplinary health professionals whose substantive training and work are within palliative care.

\section{Statistical analysis}

Data were weighted according to the 10 year age group, sex, geographic profile and country of birth using the 2006 standardised population of SA [26]. Descriptive statistics were used for respondent and patient characteristics. Multiple year comparisons were enabled by a weighting factor to ensure standardized populations were maintained [23]. Descriptive statistics were used to summarize respondent characteristics and frequency of responses. Cross tabulations using Chi square included (for the person with the life-limiting illness) principal diagnosis, (and for the caregiver) a range of demographic and caregiving characteristics. An exploratory logistic regression analysis using data from active caregivers was undertaken with factors from the cross tabulations most likely to distinguish caregiver characteristics (age of respondent as a continuous variable, highest level education, palliative care service use, Index of Socioeconomic Disadvantage (SEIFA) index) [27] and each of the unmet need domains (instrumental support, information, emotional support, finances) categories. All of the factors in the regression were biologically plausible. The model used cause of death (heart failure versus other causes) as the dependent variable to examine the caregiving needs specific to heart failure deaths. A $p$-value $<0.05$ was taken as significant.

PWAS 18.0 (SPSS Corporation Chicago Il) was used for statistical analysis. A sensitivity analysis using data with unweighted data was used to confirm the direction and magnitude of findings.

\section{Ethics and consent}

The Health Omnibus survey received State ethics committee approval in 1991, and ethical review continues annually. Verbal consent was obtained from all participants. In South Australia, informed consent can be given by anyone over the age of 15 and continuing participation in this interview was taken as continuing consent.

\section{Results}

Forty three percent of respondents (7915/18224) (unweighted) had someone close to them die in the last five years from an expected death. Approximately $5 \%$ of people who had experienced a death of someone close in the last 5 years $(4.9 \%(n=373$; weighted $)$ of all respondents) attributed this cause to HF, of whom $22.5 \%$ (84) provided active care (day-to-day or intermittent hands-on care). Active carers for people dying of HF were statistically likely to be more elderly (55.7 years; SD15.1) compared to other active caregivers (49.4 years; SD 16.6; $p=0.001)$. The period of informal caregiving lasted on average for $48.9 \pm 66.2$ months. Almost half of caregivers for HF described that the experience was worse than they expected or were unaware of what to expect. Eighty percent of HF caregivers felt that they could move on following the death of the person close to them.

Table 1 compares the respondent characteristics, place of death and uptake of palliative care services between active carers of individuals with HF and other conditions, where the cause of death was 'expected'. People with HF were statistically far less likely to access specialist palliative care services (provided by specialist palliative care providers) and likely to access palliative care services (provided by community and primary care providers) (38.1\% compared with $60.9 \%$; $p<0.0001)$. Almost half $(45.0 \%)$ reported that the person they care for was comfortable at the end of life.

Assistance with specific aspects of care was explored with active caregivers. The only difference seen between those caring for someone with HF and those with other life-limiting illnesses was in the unmet needs for hope with physical care which was twice as frequent in people with HF (28.3\% vs $14.1 \%$; $p=0.008)$. 
Table 1 Cross tabulation - respondents to the South Australian Health Omnibus who had someone close to them die in the $\mathbf{5}$ years before responding from an 'expected' death and provided a level of active hands-on care (day-to-day and intermittent care; $\mathbf{n}=\mathbf{1 5 0 4}$; weighted data)

Respondents who had someone close to them die from an expected death in the 5 years before responding who provided care for someone with ...

...heart failure ... a diagnosis other than heart failure

\section{Respondents}

Factors that do not change as caregiving is relinquished

\section{Factor}

Gender - male

Age of respondent - $65+$

Educational attainment - beyond school

Country of birth - non-English speaking

Relationship to the deceased - spouse

Factors that may change as caregiving is relinquished

Household income $\leq$ AU\$60,000

Current work status - full or part time

Region of residence - metropolitan

SEIFA index -lowest 60\%

Caregiving characteristics

Level of care - day-to-day

Length of care $-\leq 1$ year

Had enough support

Post-care factors

Time since death. $\leq 2$ years

Moving on with life - able to move on

Would care again - yes

Sought help for grief or wished they had - yes*

The deceased

Age of the deceased $>65^{*}$

Comfortable or very comfortable in the last 2 weeks of life*

Place of death - institution (hospital or hospice)*

Service factors

Palliative care service use - yes

* only asked in 2006.

\# Fisher's exact test.

** Using a Bonferroni correction, a significant $\mathrm{p}$ value should be set at 0.0025 : Fisher's Exact test used.

\begin{tabular}{|c|c|c|c|c|}
\hline Fraction & $\%$ & Fraction & $\%$ & p value ${ }^{* *}$ \\
\hline $30 / 84$ & 35.7 & $526 / 1420$ & 37.0 & 0.806 \\
\hline $23 / 84$ & 27.4 & $283 / 1420$ & 19.9 & 0.099 \\
\hline $36 / 84$ & 42.9 & $764 / 1420$ & 53.8 & 0.051 \\
\hline $12 / 84$ & 14.3 & $142 / 1420$ & 10.0 & 0.208 \\
\hline $14 / 84$ & 16.7 & $178 / 1420$ & 12.5 & 0.270 \\
\hline $50 / 68$ & 73.5 & $819 / 1240$ & 66.0 & 0.203 \\
\hline $31 / 74$ & 41.9 & $668 / 1205$ & 55.4 & 0.023 \\
\hline $52 / 83$ & 62.7 & $952 / 1420$ & 67.0 & 0.409 \\
\hline $47 / 84$ & 56.0 & $626 / 1420$ & 44.1 & 0.034 \\
\hline $35 / 84$ & 41.7 & $634 / 1420$ & 44.6 & 0.593 \\
\hline $30 / 51$ & 58.8 & $540 / 969$ & 55.7 & 0.664 \\
\hline $19 / 73$ & 26.0 & $339 / 1207$ & 28.1 & 0.704 \\
\hline $48 / 83$ & 57.8 & $803 / 1412$ & 56.9 & 0.863 \\
\hline $66 / 83$ & 79.5 & $1133 / 1408$ & 80.5 & 0.832 \\
\hline $21 / 23$ & 91.3 & 279/299 & 93.3 & 0.713 \\
\hline $11 / 30$ & 36.7 & $226 / 590$ & 38.3 & 0.857 \\
\hline $26 / 30$ & 86.7 & $428 / 592$ & 72.3 & 0.094\# \\
\hline $9 / 20$ & 45.0 & $156 / 378$ & 41.3 & 0.741 \\
\hline $20 / 29$ & 69.0 & $405 / 593$ & 68.3 & 0.940 \\
\hline $32 / 84$ & 38.1 & $865 / 1420$ & 60.9 & 0.000 \\
\hline
\end{tabular}

Table 2 reports the unmet needs identified in aggregated domains by respondents responding to two questions in 2006-2007 ( $n=5547)$ who were active caregivers for someone at the end of life at some time in the five years before responding and knew whether or not they would provide care again $(n=73)$. The need for additional instrumental assistance (physical care, symptom management, medications management) was most pronounced, but did not reach statistical significance.

Age of respondent as a continuous variable, highest level education, palliative care service use, SEIFA index and the four domains of needs (Table 2) were used in the binary regression model. The model found two significant factors that helped to explain differences between caregivers for people with $\mathrm{HF}$ and caregivers for people with other diagnoses in unmet needs at the time of death - increasing age (OR 1.02; 95\% CI 1.01 to $1.04 ; p=0.005)$ and not having access to palliative care services (OR 0.39 ; $95 \%$ CI 0.24 to $0.64 ; p=0.000$ ). For this analysis, the Hosmer and Lemeshow goodness of fit $(p=0.665)$ suggested that the model adequately fits the data and the Omnibus Tests of Model coefficients $(p=0.000)$ confirmed this. The Nagelkerke $\mathrm{R}$ square was 0.075 . 
Table 2 Unmet needs identified by respondents to the South Australian Health Omnibus in 2006, 2007 ( $n=5547)$ who had actively provided care (day-to-day or intermittent) at some time in the five years before responding for someone at the end of life and knew whether or not they would provide care again

\begin{tabular}{|c|c|c|c|c|c|}
\hline & \multicolumn{4}{|c|}{$\begin{array}{l}\text { Respondents who had someone close to them die from an } \\
\text { expected death in the } 5 \text { years before responding who died of ... }\end{array}$} & \multirow{3}{*}{$\mathrm{p}$ value } \\
\hline & & ...heart failure & & $\begin{array}{l}\text { ing illness other } \\
\text { han heart failure }\end{array}$ & \\
\hline & & $=73$ & & 1207 & \\
\hline Perceived additional support needed for: & $\mathrm{n}$ & $\%$ & $\mathbf{n}$ & $\%$ & \\
\hline physical care, symptom control, medications & 24 & 32.9 & 286 & 23.7 & 0.075 \\
\hline information about the disease progression of services available & 9 & 12.3 & 214 & 17.7 & 0.237 \\
\hline emotional, spiritual or bereavement support & 21 & 28.8 & 297 & 24.6 & 0.424 \\
\hline finances & 3 & 4.1 & 77 & 6.4 & 0.619\# \\
\hline
\end{tabular}

\# Fisher's Exact Test used.

\section{Discussion}

This study has described the characteristics of the informal HF caregiver and also identified some similarities and differences with caregivers of other conditions. The lower uptake of palliative care services by individuals with HF, compared to other conditions such as cancer, is of concern given the similar perceived needs and burden profile. This may, in practice, relate to the commonly reported challenges in determining prognosis [28]. Key findings include that in the HF population, the period of caregiving is longer than the overall mean for caregivers for people at the end of life [10].

More than $80 \%$ of active care was provided by people other than the deceased's spouse. These data reflect, in part, the changing demographics of Australian society. Of note, the rates of caregiving provided by the next generation (sons and daughters) reflect the older age of people dying from HF. This observation also has implications for issues such as time off work and broader social and economic implications of caregiving [29]. As the burden of chronic conditions including HF, continues to increase, caregiving will continue to grow as an issue [30].

The need for information about available services and knowing what to expect, is highlighted in the person's disease trajectory by this study and that the need for information is correlated with caregiving burden [31]. These data underscore the importance of providing patients and their families information of what to expect in the future [17].

\section{Study strengths}

This study provides baseline data to inform the design of interventions that may better support caregivers for people with HF. First, it is the first population based study to survey a population seeking HF caregiver needs at a whole-of-population level. The sampling process employed by the Health Omnibus allows a broadly representative sample across the whole community. Second, rather than using health care providers to survey perceptions of care and comfort, the use of independent interviewers has reduced the likelihood of a biased positive appraisal of service provision. Third, the current study is a useful proxy reflection of the people's perceptions of end of life care and is likely to relate to the deceased's experience.

\section{Limitations}

Interpretation of these data should be considered with the caveats of a retrospective, descriptive cross-sectional study and the limitations of a questionnaire items that was not subject to detailed comprehensive psychometric testing. This study used the reports of proxies to investigate the patient comfort, services use and place of death - the list is objective and reproducible - at the end of life. For symptom control in the terminal phases of a life-limiting illness, proxy reports are frequently used in palliative care research due to the difficulties encountered in research engaging people with a life-limiting illness [32]. Studies have examined the accuracy of retrospective reports by proxies, comparing reports of patients prior to death with those of their relatives after death [33]. In studies comparing concurrent prospective (pre-death) reports by patients and their relatives, family caregivers' overall symptom distress scores have been highly correlated with patients' overall scores. Families tend to rate physical symptoms more severely than patients and under-report psychological distress [34]. This is thought to occur because physical symptoms can be easier to observe than psychological distress [34]. Other factors that can affect the accuracy of proxy reports include if a caregiver is living with the person [35] and poor caregiver coping [36].

A further limitation is the post hoc nature of the survey precluded validation of HF status or description of the severity of HF. Any recall bias or response shift over time is likely to be distributed evenly between those who did and did not access specialist services. It is also important to 
note that perception of needs is related to the perception of severity, cumulative burden of symptoms and how these may change over time [37].

This study describes data from a single state, in a health system that has relatively high rates of access to specialist palliative care. The models of care and care offered differ in health settings around the world and may therefore limit the capacity to apply these findings to other health systems. There is also the potential that people may have not identified the cause of death being specifically related to HF given that caregivers' involvement in clinical consultations may, at times, be limited.

\section{Implications for practice or policy}

This study suggests that there is a need to consider more carefully how specialist palliative care services identify people whose needs are complex enough to warrant additional support as there are people currently not using the service who could potentially benefit from additional support. Ultimately, the responsibility of palliative care services should not be based on people who manage to navigate the health and social system in order to access current services, but a commitment by service providers to seek people with life-limiting illnesses and their caregivers whose complexity of needs warrant increased supports.

\section{Implications for practice}

These findings have some useful implications for HF practice. Almost 30\% of respondents reported an unmet need for support in providing physical care, and this is of particular importance to support community based care. In parallel with other studies there is an increased need for information about services and the disease trajectory and providing access to appropriate models of support for caregivers in the community. The method of recruitment, using population based sampling, is likely to access individuals who do are not recruited into studies in settings, such as HF clinics, where there is likely to be greater access to instrumental support.

\section{Implications for future research}

This study has used a retrospective, cross-sectional, population-based approach which is dependent upon recollections of bereaved respondents. Future research in this area needs to validate these findings in different health systems in prospective studies. Such research would allow a better understanding of the complexities of needs at the end of life, and how best to meet these needs across the population. Rather than face-to-face interviews, computer-assisted telephone techniques or web-based technologies can potentially be used at a population level to replicate this study in other health systems. In describing the equitable access to specialist palliative care services, the needs of people with life- limiting illnesses and their caregivers need to be considered in a more comprehensive way.

Most importantly, it will be crucial to prospectively follow the perceived needs of caregivers while in the role and correlate this with long-term caregiver health outcomes [30]. Perceived caregiver needs should be explored separately to the perceptions of the person with the life-limiting illness. For caregivers, their needs continue long into the period of time following the death and how health and social services support people who years later still have ongoing grief remains a challenge.

\section{Conclusions}

This study has contrasted similarities and differences in the caregiving experience for informal carers with HF and other conditions. As communities continue to age and less informal caregiving is available, considering the type and level of community based caregiving is likely to increase in importance. To date the majority of studies have focussed on social support [14] and the need for assistance with physical care has not been documented using a similar methodology before, and requires a focused response by service providers. These data and the work of others illustrates the complexity of the caregiving experience and the importance of assessing individuals' needs and social circumstances.

\section{Competing interests}

The authors have no conflicts of interest to disclose.

\section{Authors' contribution}

PMD: critical interpretation of the data, manuscript preparation and review; AA: study design and manuscript preparation and review; PJN critical interpretation of the data, manuscript preparation and review; KC: critical interpretation of the data, manuscript preparation and review; DCC: study design, data analysis and manuscript preparation and review. All authors read and approved the final manuscript.

\section{Author details}

${ }^{1}$ Centre for Cardiovascular and Chronic Care, Faculty of Health, University of Technology Sydney, PO Box 123, Level 7, Building 10 Jones Street, Broadway, Sydney, NSW 2007, Australia. ${ }^{2}$ Division of Medicine Oncology, Duke

University Medical Center, 3436, Durham, NC 27710, USA. ${ }^{3}$ Director \& Area Director of Palliative Care, Calvary Mater Newcastle, University of Newcastle,

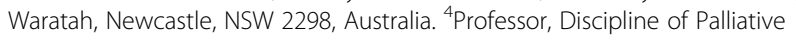
and Supportive Services, Flinders University Flinders Centre for Clinical Change, Bedford Park, 5042, South Australia, Adelaide, Australia. ${ }^{5}$ School of Nursing, Johns Hopkins University, 525 N. Wolfe Street, Baltimore, MD 21205, USA.

Received: 31 December 2012 Accepted: 27 August 2013 Published: 3 September 2013

\section{References}

1. Jaarsma T, Beattie J, Ryder M, on behalf of the Advanced Heart Failure Study Group of the HFA of the ESC, et al: Palliative care in heart failure: a position statement from the palliative care workshop of the Heart Failure Association of the European Society of Cardiology. Eur J Heart Fail 2009, 11(5):433-443.

2. Stewart S, Maclntyre K, Hole D, Capewell S, McMurray J: More 'malignant' than cancer? Five-year survival following a first admission for heart failure. Eur J Heart Fail 2001, 3:315-322.

3. Goodlin SJ: Function, failure, and frailty. J Card Fail 2010, 16(5):396 
4. Davidson PM, Paull G, Introna K, Cockburn J, Davis JM, Rees D, Gorman D, Magann L, Lafferty M, Dracup K: Integrated, collaborative palliative care in heart failure: the St. George Heart Failure Service experience 1999-2002. J Cardiovasc Nurs 2004, 19(1):68-75.

5. Johnson MJ, Parsons S, Raw J, Williams A, Daley A: Achieving preferred place of death is it possible for patients with chronic heart failure? British J cardiology 2009, 16(4):194-196.

6. Rausch SM, Baker K, Boonmee J: Sleep disturbances in caregivers of patients with end-stage congestive heart failure: part II-assess and intervene. Prog Cardiovasc Nurs 2007, 22(2):93-96.

7. Pressler SJ, Gradus-Pizlo I, Chubinski SD, Smith G, Wheeler S, Sloan R, Jung M: Family caregivers of patients with heart failure: a longitudinal study. J Cardiovasc Nurs 2013, 28(5):417-428.

8. Stewart S: Recognising the "other half" of the heart failure equation: are we doing enough for family caregivers? Eur J Heart Fail 2005, 7(4):590-591.

9. Hwang B, Luttik ML, Dracup K, Jaarsma T: Family caregiving for patients with heart failure: types of care provided and gender differences. $J$ Card Fail 2010, 16(5):398-403.

10. Aldred H, Gott M, Gariballa S: Advanced heart failure: impact on older patients and informal carers. J Adv Nurs 2005, 49(2):116-124.

11. Schulz R, Hebert RS, Dew MA, Brown SL, Scheier MF, Beach SR, Czaja SJ, Martire LM, Coon D, Langa KM, et al: Patient suffering and caregiver compassion: new opportunities for research, practice, and policy. Gerontologist 2007, 47(1):4-13.

12. Stewart S, Maclntyre K, Capewell S, McMurray JJ: Heart failure and the aging population: an increasing burden in the 21st century? Heart 2003, 89(1):49-53.

13. Gott M, Barnes S, Parker C, Payne S, Seamark D, Gariballa S, Small N: Dying trajectories in heart failure. Palliat Med 2007, 21(2):95-99.

14. Strömberg A: The situation of caregivers in heart failure and their role in improving patient outcomes. Curr Heart Fail Rep 2013, 10(3):270-275.

15. Molloy GJ, Johnston DW, Witham MD: Family caregiving and congestive heart failure. Review and analysis. Eur J Heart Fail 2005, 7(4):592-603.

16. McMillan SC, Small BJ, Haley WE, Zambroski C, Buck HG: The COPE intervention for caregivers of patients with heart failure: an adapted intervention. J Hospice \& Palliative Nurs 2013, 15(4):196-206.

17. Allen LA, Stevenson LW, Grady KL, Goldstein NE, Matlock DD, Arnold RM, Cook NR, Felker GM, Francis GS, Hauptman PJ: Decision making in advanced heart failure a scientific statement from the American Heart Association. Circulation 2012, 125(15):1928-1952.

18. Goodlin SJ, Hauptman PJ, Arnold R, Grady K, Hershberger RE, Kutner J, Masoudi F, Spertus J, Dracup K, Cleary JF: Consensus statement: palliative and supportive care in advanced heart failure. J Card Fail 2004, 10(3):200-209.

19. Burton AM, Sautter JM, Tulsky JA, Lindquist JH, Hays JC, Olsen MK, Zimmerman SI, Steinhauser KE: Burden and well-being among a diverse sample of cancer, congestive heart failure, and chronic obstructive pulmonary disease caregivers. J Pain Symptom Manag 2012, 44(3):410-420.

20. Murray SA, Kendall M, Boyd K, Worth A, Benton TF: Exploring the spiritual needs of people dying of lung cancer or heart failure: a prospective qualitative interview study of patients and their carers. Palliat Med 2004, 18(1):39-45

21. Currow D, Abernethy A, Fazekas B: Specialist palliative care needs of whole populations: a feasibility study using a novel approach. Palliat Med 2004, 18:239-247.

22. Currow DC, Agar M, Sanderson C, Abernethy AP: Populations who die without specialist palliative care: does lower uptake equate with unmet need? Palliat Med 2008, 22(1):43-50

23. Abernethy A, Currow D, Fazekas B, Luszcz M, Wheeler J, Kuchibhatla M: Specialized palliative care services are associated with improved short- and long-term caregiver outcomes. Support Care Cancer 2008, 16(6):585-597.

24. Wilson D, Wakefield M, Taylor A: The South Australian Health Omnibus survey. Health Promotion Journal Australia 1992, 2:47-49.

25. Hall J: Incremental change in the Australian health care system. Peoples Affairs 1999, 18(3):95-110.

26. Australian Bureau of Statistics: Australian demographic statistics - 3101.0 www.abs.gov.au/ausstats/abs@.nsf.

27. Currow DC, Allingham S, Bird S, Yates P, Lewis J, Dawber J, Eagar K: Referral patterns and proximity to palliative care inpatient services by level of socio-economic disadvantage. A national study using spatial analysis. BMC Health Serv Res 2012, 12(1):424.
28. Lunney JR, Lynn J, Foley DJ, Lipson S, Guralnik JM: Patterns of functional decline at the end of life. JAMA 2003, 289(18):2387-2392.

29. Australian Bureau of Statistics: Disability, ageing and carers: Summary of findings. Canberra: Commonwealth of Australia; 2004.

30. Aoun S, Kristjanson L, Currow D, Hudson P: Caregiving for the terminally ill: at what cost? Palliat Med 2005, 19(7):551-555.

31. Fried T, Bradley E, O'Leary J, Byers A: Unmet desire for caregiver-patient communication and increased caregiver burden. J Am Geriatr Soc 2005, 53(1):59-65.

32. Jordhoy MS, Kaasa S, Fayers $P$, Underland G, Ahlner-Elmqvist M: Challenges in palliative care research; recruitment, attrition and compliance: experience from a randomized controlled trial. Palliat Med 1999, 13(4):299-310

33. Addington-Hall J, McPherson C: After-death interviews with surrogates/ bereaved family members: some issues of validity. J Pain Symptom Manag 2001, 22(3):784-790.

34. McPherson CJ, Wilson KG, Lobchuk MM, Brajtman S: Family caregivers' assessment of symptoms in patients with advanced cancer: concordance with patients and factors affecting accuracy. J Pain Symptom Manag 2008, 35(1):70-82.

35. MCPherson CJ, Addington-Hall JM: Judging the quality of care at the end of life: can proxies provide reliable information? Soc Sci Med 2003, 56(1):95-109.

36. Redinbaugh EM, Baum A, DeMoss C, Fello M, Arnold R: Factors associated with the accuracy of family caregiver estimates of patient pain. J Pain Symptom Manag 2002, 23(1):31-38.

37. Davidson PM, Cockburn J, Newton PJ: Unmet needs following hospitalization with heart failure: implications for clinical assessment and program planning. J Cardiovasc Nurs 2008, 23(6):541-546.

doi:10.1186/1472-6963-13-342

Cite this article as: Davidson et al.: The caregiving perspective in heart failure: a population based study. BMC Health Services Research 2013 13:342.

\section{Submit your next manuscript to BioMed Central and take full advantage of:}

- Convenient online submission

- Thorough peer review

- No space constraints or color figure charges

- Immediate publication on acceptance

- Inclusion in PubMed, CAS, Scopus and Google Scholar

- Research which is freely available for redistribution 